\title{
Reading in the Brain Revised and Extended: Response to Comments
}

\author{
STANISLAS DEHAENE
}

\begin{abstract}
Reading in the Brain (Les neurones de la lecture, 2007) examined the origins of human reading abilities in the light of contemporary cognitive neuroscience. It argued that reading acquisition, in all cultures, recycles preexisting cortical circuits dedicated to invariant visual recognition, and that the organization of these circuits imposes strong constraints on the invention and cultural evolution of writing systems. In this article, seven years later, I briefly review new experimental evidence, particularly from brain imaging studies of illiterate adults, which indicates that reading acquisition invades culturally universal cortical circuits and competes with their prior function, including mirror-invariant visual recognition and face processing. In response to my critics, I emphasize how brain plasticity and brain constraints can be reconciled within the Bayesian perspective on learning. I also discuss the importance of a newly discovered gesture system in reading and writing. Finally, I argue that there is consistent evidence for deep cross-cultural universals in writing systems, as well as for the multiple subtypes of dyslexia that are expected given the broad set of areas recruited by the reading task.
\end{abstract}

It is a rare privilege for an author to see his work reviewed and dissected by a panel of experts. I am therefore extremely grateful to Anne Castles, Max Coltheart, Greg Downey, Naama Friedmann and Richard Menary for their thorough critical reading of Reading in the Brain (hereafter RITB).

In this reply, I first briefly review some relevant recent findings on the brain mechanisms of reading, most of which have appeared since RITB was published in 2007. Using this data as background, I then briefly respond to each of my critics.

\section{Reading in the Brain: An Update}

RITB's main thesis is that during reading acquisition, a specific region of the ventral visual occipito-temporal cortex, the visual word form area (VWFA), gets 'recycled' and specializes from the recognition of written characters. Since 2007, several fMRI studies have confirmed the central role of the VWFA in reading acquisition. In an fMRI study of adults with highly variable levels of literacy, ranging from complete illiterates to expert readers, the amount of VWFA activation to letter strings increased in direct proportion to the subjects' reading score (Dehaene, Pegado et al., 2010). Similarly, when comparing AMRI activations to written words in six-year-old children who had or had not learned to read, the main site where a difference was seen was the VWFA (Monzalvo, Fluss, Billard, Dehaene and Dehaene-Lambertz,

Address for correspondence: Inserm-CEA Cognitive Neuroimaging Unit, NeuroSpin center, CEA/SAC/DSV/I2BM Bât 145, Point Courrier 156 F-91191 Gif/Yvette, France.

Email: Stanislas.Dehaene@cea.fr 
2012). At an even younger age, in kindergarten, Brem et al. (2010) found that a few hours of exposure to the GraphoGame, a software that trains grapheme-phoneme correspondences, was sufficient for activation to emerge around the VWFA site. In adults too, training to read a new script systematically leads to changes in the VWFA, regardless of script and language (Hashimoto and Sakai, 2004; Song, Bu, $\mathrm{Hu}$, Luo and Liu, 2010; Yoncheva, Blau, Maurer and McCandliss, 2010).

An essential postulate of RITB is that the VWFA participates in a universal reading circuit, the same in all cultures and which comprises both phonological and semantic routes. fMRI studies comparing English and Chinese readers have largely confirmed this hypothesis (Booth et al., 2006; Szwed, Qiao, Jobert, Dehaene and Cohen, 2014). In fact, the reading circuit is so determined that the VWFA is activated even in congenitally blind adults when they read Braille or when they identify letters through the auditory modality using a sight-to-sound sensory substitution device (Reich, Szwed, Cohen and Amedi, 2011; Striem-Amit, Cohen, Dehaene and Amedi, 2012).

In RITB, I speculated that the surprisingly fixed localization of the VWFA arises from an intersection of two types of constraints: visual constraints that render this location particularly appropriate for discriminating small letter shapes, and connectivity constraints that interconnect it with the left superior temporal and inferior frontal language areas. In support of the first type of constraint, Szwed et al. (2011) found that configurations of line junctions, which are universally used in writing systems throughout the world (Changizi, Zhang, Ye and Shimojo, 2006), specifically promote activation in the ventral fusiform part of the visual system. In support of the second type of constraint, several papers have reported a consistent co-lateralization of the VWFA to the dominant hemisphere for spoken language and particularly to the superior temporal sulcus, suggesting a tight functional interconnection (Cai, Paulignan, Brysbaert, Ibarrola and Nazir, 2010; Pinel and Dehaene, 2009). Improvements in diffusion tensor imaging have recently allowed tracking the main fiber tracts that originate from the VWFA. As predicted, they specifically target left-hemispheric superior temporal and inferior frontal language areas, more so than other lateral or antero-posterior control sites such as the fusiform face area (Bouhali et al., 2014).

If the VWFA is recycled for reading, what is its primary function in evolution? Much of my recent research has been targeted at elucidating this point. By scanning adult illiterates with a battery of visual stimuli, we observed that prior to reading, the VWFA site is particularly responsive to faces and to checkerboards and that these responses decrease with increasing literacy (Dehaene, Pegado, et al., 2010). Remarkably, reading ability correlates with an increased displacement of face responses: activations to faces decrease in the left hemisphere and increase in the right-hemispheric fusiform face area (FFA), suggesting that a competition for cortical space between faces and written words occurs in the left occipito-temporal sulcus. These findings, which were replicated in 6-year-old and 9-year-old children (Monzalvo et al., 2012), strongly suggest that invariant visual recognition, particularly for faces, is the prior function of the VWFA and gets progressively tuned out as this cortical region specializes for letters and their combinations (for 
further evidence of cortical competition and selective pruning, see Cantlon, Pinel, Dehaene and Pelphrey, 2011; Dundas, Plaut and Behrmann, 2012; Li et al., 2013).

In RITB, I further speculated that mirror invariance (the capacity to identify an object or a face invariantly over a left-right mirror inversion) is an evolved pre-existing property of this cortical region and is progressively lost during reading acquisition, because reading imposes a fixed orientation and requires distinguishing mirror letters such as b and d. Extensive research supports this idea. First, in macaque monkeys, a patch of cortex responsive to faces and possibly homologous to the VWFA and FFA sites contains neurons whose selectivity to views of the face is entirely invariant to left-right inversion (Freiwald and Tsao, 2010). Second, using repetition suppression in humans, the AMRI response near the VWFA has been found to be mirror-invariant for objects but not for written words or letters of equivalent complexity (Dehaene, Nakamura, et al., 2010; Pegado, Nakamura, Cohen and Dehaene, 2011). Third, prior to reading, illiterate subjects present a strong mirror invariance: they are unable to distinguish symbols and other geometrical shapes from their mirrored versions (Kolinsky et al., 2011) and their capacity for mirror-image generalization, in spite of overall slower response times, appears to exceed that of literate adults (Pegado et al., 2013). These findings support the recycling model and explain why young readers frequently make mirror errors when reading and writing: mirror invariance is an evolutionary inherited function that we partially unlearn as we recycle the VWFA for reading.

In addition to confirming the role of the VWFA, recent research has also led to an extended view of the cortical changes induced by literacy. Several occipital areas, including the primary visual cortex, show an enhanced activation in literate compared to illiterate adults, even in response to non-reading stimuli such as checkerboards (Dehaene, Pegado, et al., 2010). These findings concur with fMRI evidence of an early visual tuning to letter strings (Szwed et al., 2011, 2014) and with behavioral evidence for superior visual performance in literates compared to illiterates (Szwed et al., 2012). Together, they suggest that expert reading relies heavily on early retinotopic areas (see also Sigman et al., 2005).

Another unexpected finding was that literate subjects, relative to illiterates, show enhanced activation to spoken language in the left superior temporal cortex, just posterior to the primary auditory cortex (planum temporale) (Dehaene, Pegado, et al., 2010). This result, replicated in young children (Monzalvo and Dehaene-Lambertz, 2013) and compatible with earlier fMRI findings using audio-visual sound-letter congruity (Blau, van Atteveldt, Ekkebus, Goebel and Blomert, 2009), suggests that phonological representations are changed by literacy, as further discussed below.

Finally, our understanding of the reading network has been significantly extended with the rediscovery of the crucial role of a left premotor area in the production and recognition of handwritten characters (Nakamura et al., 2012; see also Roux et al., 2009). Nakamura et al. (2012), noting that the vast majority of fMRI studies of reading used printed fonts, performed a large-scale fMRI study of the activation evoked by handwritten words in both French and Chinese readers. They discovered that a left premotor site was systematically activated, identically in both cultures, 
and contributed to the recognition of the dynamic motion pattern underlying handwritten letter shapes. This gesture recognition circuit ('reading by hand') seems to operate in parallel to the classical visual reading circuit ('reading by eye') and may be particularly essential in young children for whom manuscript writing is the norm. That both circuits exhibit similar functional properties in French and Chinese readers further supports RITB's conclusion that reading acquisition proceeds by recycling the same cerebral circuitry in all cultures.

\section{The Extent and Limits of Brain Plasticity: Response to Menary}

Menary (this issue) points to an interesting tension between two aspects of the neuronal recycling hypothesis. On the one hand, I emphasize that the child's brain is highly constrained by its genetic and epigenetic make-up (particularly its corticocortical connectivity, as noted above, but also its subcortical connectivity, local circuits, receptor distributions, cellular subtypes, etc.). I argue that these constraints place significant limits on the acquisition and even the invention of cultural tools such as reading and writing. On the other hand, as Menary points out, reading acquisition itself implies a considerable amount of neural plasticity, particularly in the human brain since only humans are able to acquire a great variety of cultural systems, each characterized by a seemingly infinite range of arbitrary choices.

To resolve this dilemma, Menary argues that, relative to other primates, the human brain evolved new mechanisms of functional plasticity in order to exploit a cognitive niche made possible by 'the scaffolding of culture and education'. He proposes that plasticity mechanisms and cultural devices co-evolved, resulting in the construction of a rich socio-cultural environment. The human brain did not evolve specifically for reading but, according to Menary, it did genetically adapt to the more general problem of quickly adjusting to diverse cultural environments. If one adopts this reasoning, he claims, 'then there is no compelling need for innate modules'.

Where do the facts stand? Since I wrote RITB, new research has amply confirmed that the brain is highly plastic and is indeed physically transformed by reading acquisition. Most striking is the fact that literate adults show enhanced fractional anisotropy in the posterior segment of the left arcuate fasciculus, a bundle of cortical connections putatively linking ventral temporal cortex (where the VWFA is located) with superior temporal and inferior parietal cortex (putatively responsible for grapheme-phoneme conversion) (Thiebaut de Schotten, Cohen, Amemiya, Braga and Dehaene, 2012). The adult brain is obviously still plastic, since those changes are found even in ex-illiterate adults who received no schooling during childhood, but became literate later on.

While this finding and many others (Carreiras et al., 2009; Castro-Caldas et al., 1999), demonstrate that plasticity is extensive even in the adult human brain, they also show that reading is systematically channeled to the same cortical circuitry. As reviewed above, reading acquisition systematically recruits the VWFA, at the same cortical location and, most strikingly, even in blind readers using Braille or 
auditory sensory substitution (Reich et al., 2011; Striem-Amit et al., 2012). This consistent localization lies at the heart of what I called the 'reading paradox' - the presence of an organized 'organ' for reading in a brain that could not have evolved for this purpose.

We are compelled to conclude that the brain is both highly plastic and highly constrained - simultaneously capable of creating an enormous variety of cultural systems and yet acquiring them within tightly defined circuits. Even beyond the reading domain, I agree with Menary that there is a remarkable tension between behavioral observations of seemingly domain-general plasticity and brain-imaging observations of constrained circuitry. Resolving this tension is perhaps one of the most significant riddles facing contemporary cognitive neuroscience. Unlike Menary, however, I doubt that the mere consideration that 'learning has a social as well as a neural dimension' will suffice to resolve this tension. Menary is right that human learning is very significantly enhanced by the uniquely human ability for teaching. A good teacher, aided by a more-or-less accurate theory of the mind of his pupils, facilitates learning by proposing a systematic progression that scaffolds the literacy acquisition process (Dehaene, Dehaene-Lambertz, Gentaz, Huron and Sprenger-Charolles, 2011). However, even non-human primates, when provided with systematic training, expand their native abilities and acquire a significant set of cultural items, including tool use, letter, digit and word recognition abilities (Diester and Nieder, 2007; Grainger, Dufau, Montant, Ziegler and Fagot, 2012; Iriki, 2005; Srihasam, Mandeville, Morocz, Sullivan and Livingstone, 2012). Furthermore, I fail to see how the scaffolding idea explains the remarkable finding that all of these cultural enhancements target specific and reproducible brain systems. Finally, I disagree that these enhancements are unbounded. Our brain certainly has a 'latent learning potential', but not a 'continual openness to radical cognitive change'.

It seems to me that the recent 'Bayesian revolution' in learning theory (Perfors, Tenenbaum, Griffiths and Xu, 2011; Tenenbaum, Kemp, Griffiths and Goodman, 2011) offers a more productive path to reconcile the apparent open-endedness of human cultural learning with the presence of tight brain constraints. The Bayesian view requires both domain-general and domain-specific mechanisms. The domain-general mechanism is the capacity to weight hypotheses according to their statistical likelihood given the input data, following the mathematical rules of Bayesian inference. A generic mechanism of Bayesian inference may be replicated throughout the brain, implemented either in the layered structure of cortex (Friston, 2005) or in the statistical properties of neuronal population coding (Ma, Beck, Latham and Pouget, 2006). But the Bayesian view also requires a collection of predefined hypotheses, within which the learning algorithm selects the most appropriate one (Kemp and Tenenbaum, 2008). As a result, Bayesian learning is not open-ended, but tightly constrained by the set of hypotheses being considered.

Reinterpreted in the light of the Bayesian framework, the neuronal recycling hypothesis boils down to assuming that each brain area, biased by minimal genetic mechanisms, codes for a dedicated set of hypotheses. The ventral visual pathway, 
for instance, may be biased to identify combinations of features forming 2D or 3D shapes (Brincat and Connor, 2004); the inferior frontal cortex ('Broca's area') may specifically learn tree structures (Musso et al., 2003); and so on. The notion that each brain area encodes an abstract hypothesis space would readily explain why the word identification stage of reading acquisition is systematically channeled to the same brain regions, even in blind subjects (Reich et al., 2011; Striem-Amit et al., 2012): shape can be conveyed in any modality, be it sight, touch or sound.

An interesting additional hypothesis is that, specifically in humans, Bayesian learning may be significantly enhanced by the capacity to consider a vastly larger set of hypotheses formed by a stochastic 'language of thought' — nested language-like expressions that recombine the pre-existing basic terms (Tenenbaum et al., 2011; T. D. Ullman, Goodman and Tenenbaum, 2012). Such a language-like system endows the Bayesian learning algorithm with an enhanced ability to acquire a variety of nested structures, including trees, maps and grammars (Kemp and Tenenbaum, 2008). In the future, it will be exciting to see if such a combinatorial mechanism can explain both the vast diversity of human cultures and the small set of abstract concepts and brain mechanisms on which they seem to be built.

\section{On the Existence of Universals of Reading: Response to Downey and to Coltheart}

Two of my critics, Downey and Coltheart, focus their remarks on the extent of cultural diversity and whether it is compatible with RITB's proposal of a tightly constrained reading circuitry.

From a cognitive anthropological standpoint, Downey presents a useful review of 'how ecological, technological and social-political factors have influenced the development of writing systems'. I am sorry if Downey misperceived RITB as 'anti-social science'. In fact, like Downey, I welcome the advent of a stronger interaction between social and neural sciences, which should ultimately result in a renewed neuro-anthropological analysis of cultural systems such as writing. Beyond visual and linguistic constraints, the reading system is clearly shaped by a variety of historical and material forces which I could not review in extenso in RITB and which Downey reviews with laudable clarity. Constraints due to the material act of writing are particularly interesting to me, given our new evidence that a premotor circuit contributes importantly to the deciphering of handwritten scripts (Nakamura et al., 2012; Roux et al., 2009). Motor constraints must have been particularly influential in the past, when handwriting was the norm. It will be interesting to see how writing evolves in the digital age.

Downey dedicates several fascinating paragraphs to Mayan writing, arguing that its reliance on face shapes was a robust feature that actually became enhanced in the course of centuries. In RITB, I briefly speculated that Mayan face-based writing would have been inefficient, due to the right-hemispheric lateralization of face recognition processes. I did not know then that, in illiterate adults and children, 
face recognition is distributed much more bilaterally in the ventral visual system, and that part if not all of the right-hemispheric lateralization of the face recognition system is a consequence of the fact that most contemporary fMRI studies scan highly literate graduate students (Dehaene, Pegado, et al., 2010; Monzalvo et al., 2012). The fact that words and faces compete for cortical territory suggests that face shapes are, in fact, a potentially appropriate stimulus for the reading circuitry. Thus, the Mayan writing system lies well within the space of constraints imposed by the human visual system and its existence fits even better with the neuronal recycling hypothesis than I thought when writing RITB. Similarly, the interesting convergence between khipu knots and Braille supports rather than dispels the idea that unrelated cultures often converge to similar devices, due to the intersection of cerebral, cognitive and anthropological constraints.

As our knowledge of these multiple constraints increases, I have no doubt that we will learn to make better predictions about the exact nature of the cross-cultural universals that they promote. Our current understanding of brain areas is incomplete and we seem to underestimate the degree of abstraction of the knowledge encoded in brain circuits (e.g. the findings from blind subjects suggest that the VWFA may be biased for abstract shapes rather than for vision per se). Correspondingly, the cross-cultural universals that are predicted in RITB may have to be revised and neuro-anthropological invariants may have to be searched at a deeper and more abstract level.

Coltheart also criticizes the proposal of cross-cultural invariants, this time from a neurological perspective. Taking a very literal interpretation of some of the diagrams in RITB, such as the hierarchy of Local Combination Detectors (Dehaene, Cohen, Sigman and Vinckier, 2005) presented as a putative neuronal model of alphabetic reading, he makes the rather trivial point that this network cannot be identical in Japanese or Hindi readers. "The very term "letterbox area", he states, 'does not apply to syllabic or logographic writing systems, since they do not have letters.' It would be hard to disagree, but I never expected that the LCD model and other such diagrams would receive such a narrow reading. Undoubtedly, the neuronal architecture for invariant visual recognition, which is getting increasingly understood (DiCarlo, Zoccolan and Rust, 2012), must adapt to the specific task of recognizing letters, Kanji or Hindi characters. Importantly, its key aspects are being integrated into massive neural network simulations that solve actual image recognition tasks with a performance approaching that of human observers (Isik, Leibo and Poggio, 2012; Serre, Oliva and Poggio, 2007). It will therefore soon be possible to simulate how these networks behave when confronted with the evolutionarily novel task of recognizing written words in different scripts. My goal in RTIB was to incite neural modelers to run such simulations of reading acquisition and, conversely, to incite reading researchers to think of how their models could be implemented within the existing constraints of the visual system. It would be very exciting to start with a generic model of invariant visual recognition, such as Poggio's HMAX or a variant thereof and to see how such a system recycles to the visual recognition of French, English, Japanese or Hindi words. I have no 
doubt that, when we do so, we will discover that receptive field and connectivity constraints play out in a very similar manner in all of these languages.

The neuronal recycling model makes a large number of specific predictions for cross-cultural universals. According to Coltheart, we already know that these predictions are violated. I disagree. Specifically, I argue for the following points:

(1) the visual system forces writing systems to display words using a compact foveal shape, otherwise they could not be identified with sufficient speed and precision. Coltheart retorts that very long words exist, for instance in Finnish. This is unquestionably, incontrovertibly true, but irrelevant. My prediction is specifically that such words will not be analyzed as a single unit, but will require multiple fixations or, at the very least, multiple attentional movements. As a result, they will exhibit a strong word length effect. I know of no evidence that goes against this idea. On the contrary, in both English and French, words that comprise between 3 and 8 letters are recognized equally quickly by adult readers, but words with more than 8 letters begin to exhibit a very significant length effect (New, Ferrand, Pallier and Brysbaert, 2006).

(2) Written word recognition relies on the same neuronal hierarchy of nested elementary shapes which is used for object or face recognition (Brincat and Connor, 2004; DiCarlo et al., 2012; Serre et al., 2007; S. Ullman, 2007). Therefore, all writing systems should make use of nested combinations of elementary shapes (e.g. letters). Coltheart retorts that there is no obvious inventory of component shapes in Mayan, Kanji or Chinese writing. I predict that there is, i.e. that the visual system decomposes Mayan figures (into eyes, mouth, hairstyle etc.) and Kanji characters (into frequent groups of strokes) and that writers and readers are sensitive to their presence. Even a cursory look at the inventory of Mayan glyphs (http://www.omniglot.com/writing/mayan.htm) reveals that they are often composed of reproducible subparts (e.g. compare the characters for cha, se, tse and tze). Most importantly, contrary to what Coltheart claims, Chinese characters are not indivisible. Many of them can be described as a hierarchy of strokes forming groups called 'radicals' that, in many cases, convey distinct semantic and phonetic information. Furthermore, these subunits are extracted by the visual system, as demonstrated by the fact that repetition priming can be obtained when these radicals are repeated in otherwise unrelated Chinese words (Ding, Peng and Taft, 2004). Phonetic radicals convey statistical information about the pronunciation of Chinese characters and expert Chinese readers make use of this sublexical route (Kuo et al., 2004; Lee et al., 2004). Such data support RITB's proposal that reading systems universally makes use of the visual system's capacity to decompose visual shapes into subparts, although the exact manner in which this is accomplished varies across cultures. 
(3) Alphabetic characters consist of approximately three strokes. Here I was citing a discovery by Changizi and Shimojo (2005), which Coltheart does not seem to contest. The observation that Chinese characters consist of many more strokes is irrelevant. I do not expect a single Chinese character and a single letter to be recognized at the same stage of the visual hierarchy. The prediction here is that any writing system (including Chinese characters) relies on a hierarchy of subparts and that one level of this hierarchy should match the visual complexity of a single letter, i.e. about 3 strokes. Further quantitative analysis is obviously needed, but the graphic elements such as 兀,力,十, ᄃ,土,大, 寸, etc., whose combinations form the basis of many Chinese characters (e.g. 坎,村 or 努), seem to fit with this proposal. More generally, I speculate that, within the visual system, when transitioning from one level of the neuronal hierarchy to the next, the type of preferred subparts will consist in a combination of 2-4 subparts at the immediately lower level. There is direct evidence that the shape preference of many infero-temporal neurons is captured by a combination of 2-4 elementary strokes (Brincat and Connor, 2004, 2006).

(4) All writing systems convert the temporal dimension of speech into the spatial dimension of the written page. As a result, reading operates as a progressive movement of spatial attention and the eyes (with occasional jumps to the next line). I know of only one exception to this rule: when Chinese writing was introduced in Korea and Japan, words were written with diacritical marks that indicated how they should be reordered according to Japanese or Korean syntax (thus putting a severe burden on the reader). It is only because Coltheart takes an exceedingly narrow perspective on my proposal that he sees the co-existence of multiple reading directions (in ancient Greek boustrophedon, or in Japanese) as a problem-in all of these cases, each line of text imposes a precise correspondence between the position of written characters and the order of the corresponding speech sounds.

(5) All writing systems capitalize on the size and location invariance provided by the ventral visual pathway. Coltheart's counter-examples are thought provoking, but do not introduce major violations. The fact that Hebrew and Arabic use different letter shapes in word-initial, medial and word-final positions is intriguing, but does not mean that location invariance is absent in these languages: because word length and eye fixation vary, all of these letter shapes appear at a variety of retinal locations during reading and this is transparent to the reader (and writer). Likewise, the distinction made possible by upper- and lower-case letters in our alphabet (e.g. between Smith and smith) only shows that relative size is occasionally important, but it should not hide that an English reader recognizes these words efficiently across a broad range of absolute sizes (Chung, Mansfield and Legge, 1998; Legge, Pelli, Rubin and Schleske, 1985; Pelli, Burns, Farell and MoorePage, 2006). It should also be noted that Coltheart's examples are rare exceptions to an otherwise stable cross-cultural pattern. 


\section{Dyslexia and its Subtypes: Response to Castles and Friedmann}

My last critics, Castles and Friedmann, focus specifically on RITB's Chapter 6, dedicated to developmental dyslexia. There, I tried to summarize, as honestly as I could, the state of the art in a literature to which I contributed little. My goal was to present the current consensus, which is that phonological deficits are a dominant factor in the majority of cases of dyslexia. I am sorry if I over-stated the case (in my mind, 'the majority' simply means 'more than 50\%', not 'virtually all'), but it seemed important to state the behavioral, brain-imaging and genetic evidence clearly because, in France at least, an abundant variety of self-pronounced experts still propose a paraphernalia of non-scientific approaches and cures to dyslexia, while others, often from a psychoanalytic stance, deny that it even exists. However, throughout this chapter, I did not hide the difficulties with this mono-causal story, nor my own skepticism at the idea that this would be the sole explanation for all developmental reading disorders.

In fact, when writing RITB, I was heavily influenced by Castles and Coltheart's (2004) critical review, where they argued that 'no study has provided unequivocal evidence that there is a causal link from competence in phonological awareness to success in reading and spelling acquisition' and that many of the observed phonological deficits could, in fact, be a consequence rather than a cause of early differences in literacy. Two recent studies of literacy acquisition from my laboratory (Dehaene, Pegado, et al., 2010; Monzalvo and Dehaene-Lambertz, 2013) support this point of view by showing that, in normally developing six-years-olds and adults, literacy acquisition strongly enhances the activation of the left planum temporale during speech listening, at a site thought to encode phonetic categories (e.g. Chang et al., 2010). The causal role of phonological impairments is further weakened by a recent study which uses multivariate pattern analysis of $\mathrm{fMRI}$ data to demonstrate a normal phonological representation in the superior temporal plane of dyslexic adults (Boets et al., 2013). In that study, the deficit that remains in adults is a temporo-frontal disconnection, suggestive of an impaired access to phonology-yet even that could still be a consequence rather than a cause of impaired reading.

In RITB, I tried to explain the complexity of reading circuits and therefore the great likelihood that many of its components, if failing, could induce a developmental reading deficit. I purposely devoted many pages of Chapter 7 to a remarkable case of visual dyslexia (McCloskey and Rapp, 2000), which is too rarely cited, in my opinion and which can only be explained by an anomaly within the visual mechanisms of mirror invariance. I therefore find myself in agreement with most of Castles and Friedmann's arguments against the idea that there a single kind of dyslexia, caused solely by a phonological deficit, and I deeply appreciated their interesting review of the various types of developmental reading disorders, including surface dyslexia, letter position dyslexia and attentional dyslexia - thus adding complexity to the admittedly schematic picture of developmental dyslexia that I painted in RITB.

Much of my recent research points to the complexity of the visual components of reading. I therefore suspect that visual factors have been insufficiently studied in 
reading impairments. In fact, my laboratory obtained direct evidence for a severe disorganization of the ventral visual system in nine-year old dyslexic children (Monzalvo et al., 2012), although again we cannot know whether this is a cause or a consequence of abnormal reading acquisition.

Particularly interesting to me is the possibility of a strong contribution of the parietal spatial attention circuit to reading development. We know that this system is active during reading of degraded words in adults, where its activation is accompanied by a serial reading strategy characterized by a strong effect of word length on reading times (Cohen, Dehaene, Vinckier, Jobert and Montavont, 2008). It is likely to be particularly important in childhood, given that early reading is characterized by a strong effect of word length, which progressively vanishes as automatization sets in (Aghababian and Nazir, 2000; Zoccolotti et al., 2005). In the light of recent evidence suggesting that a subcategory of dyslexic children may suffer from specific impairments to the posterior parietal visuospatial attention system (Facoetti, Paganoni, Turatto, Marzola and Mascetti, 2000; Peyrin et al., 2012; Peyrin, Demonet, N'Guyen-Morel, Le Bas and Valdois, 2011; Vidyasagar and Pammer, 2010) and that pre-reading attention ability predicts reading scores (Franceschini, Gori, Ruffino, Pedrolli and Facoetti, 2012), it seems high time to re-examine whether some dyslexic disorders could be alleviated by attention training (see also Franceschini et al., 2013; Tang and Posner, 2009).

\section{Conclusion}

I am grateful to my critics for pointing to areas where Reading in the Brain could be improved. Most science books age fast. If I had time and space to rewrite and extend the book today, I would emphasize the role of a premotor gesture system in handwritten recognition and its likely influence on the evolution of writing, together with other political and historical forces; I would dedicate more space to the illiterate brain and to our greatly enhanced understanding of how literacy acquisition changes it; and I would describe the various subtypes of dyslexia more carefully. None of these points detract from my main thesis, which is that our reading and writing systems are strongly constrained by the organization of the brain circuits that we recycle as we acquire literacy.

Collège de France and INSERM-CEA Cognitive Neuroimaging Unit

\section{References}

Aghababian, V. and Nazir, T. A. 2000: Developing normal reading skills: aspects of the visual processes underlying word recognition. Journal of Experimental Child Psychology, 76(2), $123-50$. 
Blau, V., van Atteveldt, N., Ekkebus, M., Goebel, R. and Blomert, L. 2009: Reduced neural integration of letters and speech sounds links phonological and reading deficits in adult dyslexia. Current Biology, 19(6), 503-8.

Boets, B., Op de Beeck, H. P., Vandermosten, M., Scott, S. K., Gillebert, C. R., Mantini, D., Ghesquiere, P. et al. 2013: Intact but less accessible phonetic representations in adults with dyslexia. Science, 342(6163), 1251-4.

Booth, J. R., Lu, D., Burman, D. D., Chou, T.-L., Jin, Z., Peng, D.-L., Liu, L. et al. 2006 : Specialization of phonological and semantic processing in Chinese word reading. Brain Research, 1071, 197-207. doi:10.1016/j.brainres.2005.11.097

Bouhali, F., Thiebaut de Schotten, M., Pinel, P., Poupon, C., Mangin, J. F., Dehaene, S. and Cohen, L. 2014: Anatomical connections of the visual word form area. Journal of Neuroscience, submitted.

Brem, S., Bach, S., Kucian, K., Guttorm, T. K., Martin, E., Lyytinen, H., Richardson, U. et al. 2010: Brain sensitivity to print emerges when children learn letter-speech sound correspondences. Proceedings of the National Academy of Sciences USA, 107(17), 7939-44.

Brincat, S. L. and Connor, C. E. 2004: Underlying principles of visual shape selectivity in posterior inferotemporal cortex. Nature Neuroscience, 7(8), 880-6.

Brincat, S. L. and Connor, C. E. 2006: Dynamic shape synthesis in posterior inferotemporal cortex. Neuron, 49, 17-24.

Cai, Q., Paulignan, Y., Brysbaert, M., Ibarrola, D. and Nazir, T. A. 2010: The left ventral occipito-temporal response to words depends on language lateralization but not on visual familiarity. Cerebral Cortex, 20, 1153-63.

Cantlon, J. F., Pinel, P., Dehaene, S. and Pelphrey, K. A. 2011: Cortical representations of symbols, objects and faces are pruned back during early childhood. Cerebral Cortex, 21, 191-9.

Carreiras, M., Seghier, M. L., Baquero, S., Estevez, A., Lozano, A., Devlin, J. T. and Price, C. J. 2009: An anatomical signature for literacy. Nature, 461(7266), 983-6.

Castles, A. and Coltheart, M. 2004: Is there a causal link from phonological awareness to success in learning to read? Cognition, 91, 77-111.

Castro-Caldas, A., Miranda, P. C., Carmo, I., Reis, A., Leote, F., Ribeiro, C. and Ducla-Soares, E. 1999: Influence of learning to read and write on the morphology of the corpus callosum. European Journal of Neurology, 6, 23-8.

Chang, E. F., Rieger, J. W., Johnson, K., Berger, M. S., Barbaro, N. M. and Knight, R. T. 2010: Categorical speech representation in human superior temporal gyrus. Nature Neuroscience, 13(11), 1428-32.

Changizi, M. A. and Shimojo, S. 2005: Character complexity and redundancy in writing systems over human history. Proceedings of the Royal Society B: Biological Sciences, 272(1560), 267-75. 
Changizi, M. A., Zhang, Q., Ye, H. and Shimojo, S. 2006: The structures of letters and symbols throughout human history are selected to match those found in objects in natural scenes. The American Naturalist, 167(5), E117-39.

Chung, S. T., Mansfield, J. S. and Legge, G. E. 1998: Psychophysics of reading. XVIII. The effect of print size on reading speed in normal peripheral vision. Vision Research, 38(19), 2949-62.

Cohen, L., Dehaene, S., Vinckier, F., Jobert, A. and Montavont, A. 2008: Reading normal and degraded words: contribution of the dorsal and ventral visual pathways. Neuroimage, 40, 353-66.

Dehaene, S., Cohen, L., Sigman, M. and Vinckier, F. 2005: The neural code for written words: a proposal. Trends in Cognitive Sciences, 9(7), 335-41.

Dehaene, S., Dehaene-Lambertz, G., Gentaz, E., Huron, C. and Sprenger-Charolles, L. 2011: Apprendre à lire: Des sciences cognitives à la salle de classe. Paris: Editions Odile Jacob.

Dehaene, S., Nakamura, K., Jobert, A., Kuroki, C., Ogawa, S. and Cohen, L. 2010a: Why do children make mirror errors in reading? Neural correlates of mirror invariance in the visual word form area. Neuroimage, 49(2), 1837-48.

Dehaene, S., Pegado, F., Braga, L. W., Ventura, P., Nunes Filho, G., Jobert, A., Cohen, L. et al. 2010b: How learning to read changes the cortical networks for vision and language. Science, 330(6009), 1359-64.

DiCarlo, J. J., Zoccolan, D. and Rust, N. C. 2012: How does the brain solve visual object recognition? Neuron, 73(3), 415-34.

Diester, I. and Nieder, A. 2007: Semantic associations between signs and numerical categories in the prefrontal cortex. PLoS Biology, 5(11), e294.

Ding, G., Peng, D. and Taft, M. 2004: The nature of the mental representation of radicals in Chinese: a priming study. Journal of Experimental Psychology: Learning, Memory, and Cognition, 30(2), 530-9.

Dundas, E. M., Plaut, D. C. and Behrmann, M. 2012: The joint development of hemispheric lateralization for words and faces. Journal of Experimental Psychology: General, 142, 348-58.

Facoetti, A., Paganoni, P., Turatto, M., Marzola, V. and Mascetti, G. G. 2000: Visual-spatial attention in developmental dyslexia. Cortex, 36, 109-23.

Franceschini, S., Gori, S., Ruffino, M., Pedrolli, K. and Facoetti, A. 2012: A causal link between visual spatial attention and reading acquisition. Current Biology, 22(9), 814-819.

Franceschini, S., Gori, S., Ruffino, M., Viola, S., Molteni, M. and Facoetti, A. 2013: Action video games make dyslexic children read better. Current Biology, 23(6), 462-6.

Freiwald, W. A. and Tsao, D. Y. 2010: Functional compartmentalization and viewpoint generalization within the macaque face-processing system. Science, 330(6005), $845-51$. 
Friston, K. 2005: A theory of cortical responses. Philosophical Transactions of the Royal Society B: Biological Sciences, 360(1456), 815-36. doi:10.1098/rstb.2005.1622

Grainger, J., Dufau, S., Montant, M., Ziegler, J. C. and Fagot, J. 2012: Orthographic processing in Baboons (Papio papio). Science, 336(6078), 245-8.

Hashimoto, R. and Sakai, K. L. 2004: Learning letters in adulthood: direct visualization of cortical plasticity for forming a new link between orthography and phonology. Neuron, 42(2), 311-22.

Iriki, A. 2005: A prototype of Homo-Faber: a silent precursor of human intelligence in the tool-using monkey brain. In S. Dehaene, J. R. Duhamel, M. Hauser and G. Rizzolatti (eds), From Monkey Brain to Human Brain. Cambridge, MA: MIT Press, pp. $133-57$.

Isik, L., Leibo, J. Z. and Poggio, T. 2012: Learning and disrupting invariance in visual recognition with a temporal association rule. Frontiers in Computational Neuroscience, 6. doi:10.3389/fncom.2012.00037

Kemp, C. and Tenenbaum, J. B. 2008: The discovery of structural form. Proceedings of the National Academy of Sciences USA, 105(31), 10687-92.

Kolinsky, R., Verhaeghe, A., Fernandes, T., Mengarda, E. J., Grimm-Cabral, L. and Morais, J. 2011: Enantiomorphy through the looking-glass: literacy effects on mirror-image discrimination. Journal of Experimental Psychology: General, 140(2), $210-38$.

Kuo, W. J., Yeh, T. C., Lee, J. R., Chen, L. F., Lee, P. L., Chen, S. S., Ho, L. T., Hung, D. L., Tzeng, O. J. and Hsieh, J. C. 2004: Orthographic and phonological processing of Chinese characters: an fMRI study. Neuroimage, 21, 1721-31.

Lee, C. Y., Tsai, J. L., Kuo, W. J., Yeh, T. C., Wu, Y. T., Ho, L. T. et al. 2004 :Neuronal correlates of consistency and frequency effects on Chinese character naming: an event-related fMRI study, Neuroimage, 23, 1235-45.

Legge, G. E., Pelli, D. G., Rubin, G. S. and Schleske, M. M. 1985: Psychophysics of reading. I. Normal vision. Vision Research, 25(2), 239-52.

Li, S., Lee, K., Zhao, J., Yang, Z., He, S. and Weng, X. 2013: Neural competition as a developmental process: early hemispheric specialization for word processing delays specialization for face processing. Neuropsychologia, 51(5), 950-9.

Ma, W. J., Beck, J. M., Latham, P. E. and Pouget, A. 2006: Bayesian inference with probabilistic population codes. Nature Neuroscience, 9(11), 1432-8.

McCloskey, M. and Rapp, B. 2000: A visually based developmental reading deficit. Journal of Memory and Language, 43, 157-81.

Monzalvo, K. and Dehaene-Lambertz, G. 2013: How reading acquisition changes children's spoken language network. Brain and Language, 127(3), 356-65.

Monzalvo, K., Fluss, J., Billard, C., Dehaene, S. and Dehaene-Lambertz, G. 2012: Cortical networks for vision and language in dyslexic and normal children of variable socio-economic status. Neuroimage, 61, 258-74. 
Musso, M., Moro, A., Glauche, V., Rijntjes, M., Reichenbach, J., Buchel, C. and Weiller, C. 2003: Broca's area and the language instinct. Nature Neuroscience, 6(7), 774-81.

Nakamura, K., Kuo, W. J., Pegado, F., Cohen, L., Tzeng, O. J. and Dehaene, S. 2012: Universal brain systems for recognizing word shapes and handwriting gestures during reading. Proceedings of the National Academy of Sciences USA, 109(50), 20762-7.

New, B., Ferrand, L., Pallier, C. and Brysbaert, M. 2006: Reexamining the word length effect in visual word recognition: new evidence from the English Lexicon Project. Psychonomic Bulletin and Review, 13, 45-52.

Pegado, F., Nakamura, K., Braga, L. W., Ventura, P., Filho, G. N., Pallier, C., Dehaene, S. et al. 2013: Literacy breaks mirror invariance for visual stimuli: a behavioral study with adult illiterates. Journal of Experimental Psychology. General. doi:10.1037/a0033198

Pegado, F., Nakamura, K., Cohen, L. and Dehaene, S. 2011: Breaking the symmetry: mirror discrimination for single letters but not for pictures in the Visual Word Form Area. Neuroimage, 55(2), 742-9.

Pelli, D. G., Burns, C. W., Farell, B. and Moore-Page, D. C. 2006: Feature detection and letter identification. Vision Research, 46(28), 4646-74.

Perfors, A., Tenenbaum, J. B., Griffiths, T. L. and Xu, F. 2011: A tutorial introduction to Bayesian models of cognitive development. Cognition, 120(3), 302-21.

Peyrin, C., Demonet, J. F., N'Guyen-Morel, M. A., Le Bas, J. F. and Valdois, S. 2011: Superior parietal lobule dysfunction in a homogeneous group of dyslexic children with a visual attention span disorder. Brain and Language, 118(3), 128-38.

Peyrin, C., Lallier, M., Demonet, J. F., Pernet, C., Baciu, M., Le Bas, J. F. and Valdois, S. 2012: Neural dissociation of phonological and visual attention span disorders in developmental dyslexia: FMRI evidence from two case reports. Brain and Language, 120(3), 381-94.

Pinel, P. and Dehaene, S. 2009: Beyond hemispheric dominance: brain regions underlying the joint lateralization of language and arithmetic to the left hemisphere. Journal of Cognitive Neuroscience, 22, 48-66.

Reich, L., Szwed, M., Cohen, L. and Amedi, A. 2011: A ventral visual stream reading center independent of visual experience. Current Biology, 21(5), 363-8.

Roux, F. E., Dufor, O., Giussani, C., Wamain, Y., Draper, L., Longcamp, M. and Demonet, J. F. 2009: The graphemic/motor frontal area Exner's area revisited. Annals of Neurology, 66(4), 537-45.

Serre, T., Oliva, A. and Poggio, T. 2007: A feedforward architecture accounts for rapid categorization. Proceedings of the National Academy of Sciences USA, 104(15), 6424-9.

Sigman, M., Pan, H., Yang, Y., Stern, E., Silbersweig, D. and Gilbert, C. D. 2005 : Top-down reorganization of activity in the visual pathway after learning a shape identification task. Neuron, 46(5), 823-35. 
Song, Y., Bu, Y., Hu, S., Luo, Y. and Liu, J. 2010: Short-term language experience shapes the plasticity of the visual word form area. Brain Research, 1316, 83-91.

Srihasam, K., Mandeville, J. B., Morocz, I. A., Sullivan, K. J. and Livingstone, M. S. 2012: Behavioral and anatomical consequences of early versus late symbol training in macaques. Neuron, 73(3), 608-19.

Striem-Amit, E., Cohen, L., Dehaene, S. and Amedi, A. 2012: Reading with sounds: sensory substitution selectively activates the visual word form area in the blind. Neuron, 76(3), 640-52.

Szwed, M., Dehaene, S., Kleinschmidt, A., Eger, E., Valabregue, R., Amadon, A. and Cohen, L. 2011: Specialization for written words over objects in the visual cortex. Neuroimage, 56, 330-44.

Szwed, M. Ventura, P., Querido, L., Cohen, L. and Dehaene, S. 2012: Reading acquisition enhances an early visual process of contour integration. Developmental Science, 15, 139-49.

Szwed, M., Qiao, E., Jobert, A., Dehaene, S. and Cohen, L. 2014: Effects of literacy in early visual and occipitotemporal areas of Chinese and French readers. Journal of Cognitive Neuroscience, in press.

Tang, Y.-Y. and Posner, M. I. 2009: Attention training and attention state training. Trends in Cognitive Sciences, 13(5), 222-7.

Tenenbaum, J. B., Kemp, C., Griffiths, T. L. and Goodman, N. D. 2011: How to grow a mind: statistics, structure and abstraction. Science, 331(6022), 1279-85.

Thiebaut de Schotten, M., Cohen, L., Amemiya, E., Braga, L. W. and Dehaene, S. 2012: Learning to read improves the structure of the arcuate fasciculus. Cerebral Cortex, 24, 989-95.

Ullman, S. 2007: Object recognition and segmentation by a fragment-based hierarchy. Trends in Cognitive Science, 11(2), 58-64.

Ullman, T. D., Goodman, N. D. and Tenenbaum, A. 2012: Theory learning as stochastic search in the language of thought. Cognitive Development, 27, 455-80.

Vidyasagar, T. R. and Pammer, K. 2010: Dyslexia: a deficit in visuo-spatial attention, not in phonological processing. Trends in Cognitive Science, 14(2), 57-63.

Yoncheva, Y. N., Blau, V. C., Maurer, U. and McCandliss, B. D. 2010: Attentional focus during learning impacts N170 ERP responses to an artificial script. Developmental Neuropsychology, 35(4), 423-45.

Zoccolotti, P., De Luca, M., Di Pace, E., Gasperini, F., Judica, A. and Spinelli, D. 2005: Word length effect in early reading and in developmental dyslexia. Brain and Language, 93(3), 369-73. 